\title{
Associations Between Daily Affective Instability and Connectomics in Functional Subnetworks in Remitted Patients with Recurrent Major Depressive Disorder
}

\author{
Michelle N Servaas ${ }^{*, 1,2,3}$, Harriëtte Riese ${ }^{2}$, Remco J Renken ${ }^{3}$, Marieke Wichers ${ }^{2}$, Jojanneke A Bastiaansen ${ }^{2,4}$, \\ Caroline A Figueroa ${ }^{5}$, Hanneke Geugies ${ }^{1,3}$, Roel JT Mocking ${ }^{5}$, Linda Geerligs ${ }^{6}$, Jan-Bernard C Marsman ${ }^{3}$, \\ André Aleman ${ }^{3,7}$, Aart H Schene ${ }^{6,8}$, Robert A Schoevers ${ }^{1,2}$ and Henricus G Ruhé ${ }^{1,2,5,9}$ \\ 'Department of Psychiatry, Mood and Anxiety Disorders, University Medical Center Groningen, University of Groningen, Groningen, The \\ Netherlands; ${ }^{2}$ Department of Psychiatry, University Medical Center Groningen, Interdisciplinary Center for Psychopathology and Emotion \\ Regulation, University of Groningen, Groningen, The Netherlands; ${ }^{3}$ Department of Neuroscience, University of Groningen, University Medical Center \\ Groningen, Neuroimaging Center, Groningen, The Netherlands; ${ }^{4}$ Department of Education and Research, Friesland Mental Health Care Services, \\ Leeuwarden, The Netherlands; ${ }^{5}$ Department of Psychiatry, Academic Medical Center, University of Amsterdam, Amsterdam, The Netherlands; \\ ${ }^{6}$ Donders Institute for Brain, Cognition and Behavior, Radboud University, Nijmegen, The Netherlands; ' ${ }^{7}$ Department of Psychology, University of \\ Groningen, The Netherlands; ${ }^{8}$ Department of Psychiatry, Radboud University Medical Center, Nijmegen, The Netherlands; ${ }^{9}$ Department of \\ Psychiatry, University of Oxford, Warnford Hospital, Oxford, UK
}

\begin{abstract}
Remitted patients with major depressive disorder (rMDD) often report more fluctuations in mood as residual symptomatology. It is unclear how this affective instability is associated with information processing related to the default mode (DMS), salience/reward (SRS), and frontoparietal (FPS) subnetworks in rMDD patients at high risk of recurrence (rrMDD). Sixty-two unipolar, drug-free rrMDD patients $(\geqslant 2$ MDD episodes) and $4 \mathrm{I}$ healthy controls ( $\mathrm{HCs}$ ) were recruited. We used experience sampling methodology to monitor mood/ cognitions ( 10 times a day for 6 days) and calculated affective instability using the mean adjusted absolute successive difference. Subsequently, we collected resting-state functional magnetic resonance imaging data and performed graph theory to obtain network metrics of integration within (local efficiency) the DMS, SRS, and FPS, and between (participation coefficient) these subnetworks and others. In rrMDD patients compared with HCs, we found that affective instability was increased in most negative mood/cognition variables and that the DMS had less connections with other subnetworks. Furthermore, we found that rrMDD patients, who showed more instability in feeling down and irritated, had less connections between the SRS and other subnetworks and higher local efficiency coefficients in the FPS, respectively. In conclusion, rrMDD patients, compared with HCs, are less stable in their negative mood and these dynamics are related to differences in information processing within- and between-specific functional subnetworks. These results are a first step to gain a better understanding of how mood fluctuations in real life are represented in the brain and provide insights into the vulnerability profile of MDD.
\end{abstract}

Neuropsychopharmacology (2017) 42, 2583-2592; doi:10.1038/npp.2017.65; published online 10 May 2017

\section{INTRODUCTION}

Generally, major depressive disorder (MDD) has an intermittent course in which remission and recovery are often followed by relapse and recurrence, respectively (Steinert et al, 2014). The most important predictors of relapse/ recurrence are the number of previous episodes and the presence of residual symptomatology after recovery from

*Correspondence: Dr MN Servaas, Department of Neuroscience, University of Groningen, University Medical Center Groningen, Neuroimaging Center, PO Box 196, Groningen 9700 AD, The Netherlands, Tel: +3I 5036 | 6404, Fax: +3I 50363 8875, E-mail: m.n.servaas@umcg.nl

Received 23 November 2016; revised 22 March 2017; accepted 24 March 2017; accepted article preview online 31 March 2017
MDD (Hardeveld et al, 2010). To gain insight into vulnerability for MDD, it is important to investigate residual symptomatology (Fava and Visani, 2008) and its associated neurobiological correlates (De Raedt and Koster, 2010; Marchetti et al, 2012) in remitted patients with recurrent MDD as they are at high risk for another episode.

Residual symptoms, often reported by remitted MDD (rMDD) patients, are alterations in mood (Fava and Visani, 2008; Aan het Rot et al, 2012). These can be monitored on a daily basis using the experience sampling method (ESM). In ESM, individuals fill out short self-report questionnaires on affect, physical status, and context several times a day (Trull et al, 2008). Prior ESM studies showed that rMDD patients report on average higher negative mood and lower positive mood compared with healthy controls (HCs) (Knowles et al, 
2007; van Winkel et al, 2015). Furthermore, rMDD patients showed higher reactivity in mood to daily (Husky et al, 2009; O'Hara et al, 2014) and social (van Winkel et al, 2015) stressors compared with HCs. These latter results may point to more affective instability (higher variability and lower temporal dependency in mood ratings; Trull et al, 2008) in rMDD patients, as reactivity of negative affect to negative external events has been positively related to instability of negative affect (Thompson et al, 2012). An indication for the latter in rMDD patients has been observed by Thompson et al (2011), who showed increased affective instability in these patients compared with individuals without a lifetime history of MDD using the borderline personality disorder module of the Personality Disorder Interview-IV (PDI-IV; Widiger et al, 1995). Furthermore, a recent meta-analysis has shown an association between lower psychological wellbeing and higher affective instability (specifically negative emotions) in MDD patients (Houben et al, 2015). In the current study, using ESM, we investigated whether high affective instability is characteristic of remitted recurrent MDD (rrMDD) patients, who are at high risk of relapse/ recurrence.

Moreover, neuroimaging studies have demonstrated alterations in neural correlates related to emotion processing in MDD patients (Groenewold et al, 2013). A recently adopted perspective, wherein the brain is viewed as a complex network supporting the integration and segregation of information processing, has provided further insights into the large-scale abnormalities in topological network organization in these patients (Gong and He, 2015). Using graph theory, the brain is defined as a graph consisting of nodes (ie, brain regions, voxels) and edges (ie, connections between nodes) on which global/local (eg, efficiency) and nodal (eg, degree) metrics can be calculated (Rubinov and Sporns, 2010). To date, two white-matter structural connectomic studies have been performed in rMDD patients, showing alterations in metrics calculated on nodes that are part of the DMS (self-reflection), SS (negative attention bias/reward), and FPS (cognitive control) subnetwork (Bai et al, 2012; Qin et al, 2015). Alterations in these subnetworks have been related to symptoms in (r)MDD patients (Hamilton et al, 2013; Jacobs et al, 2014; Mulders et al, 2015). Studies on the functional network organization have not yet been performed in rMDD. However, this would allow researchers to investigate whether disturbances in the integration and segregation of information processing in the abovementioned subnetworks-as observed in MDD patients (Gong and He, 2015) - are characteristic of rrMDD patients, who are at high risk of relapse/recurrence.

An interesting property of resting-state functional magnetic resonance imaging (rs-fMRI) data, on which graph theory is performed, is that it exhibits experience-dependent changes over time (Sporns, 2012). During rest, the connectome seems to rehearse mental states via spontaneous neural activity by reactivating genetically and experientially modified pathways (Sporns, 2012). Hence, it is of specific interest to combine rs-fMRI with ESM, which has not yet been done. This would allow us to investigate whether alterations in affective instability are associated with alterations in connectivity within (ie, segregation of information processing) and between (ie, integration of information processing) specific functional subnetworks. In the current study, we hypothesized to find, in rrMDD patients compared with HCs, (i) increased affective instability in mood/ cognition variables, specifically variables related to negative affect, (ii) alterations in network metrics capturing connectivity within (ie, local efficiency) the DMS, SS, and FPS, and between (ie, participation coefficient) these subnetworks and others (Servaas et al, 2015), (iii) increased affective instability in mood/cognition variables to be associated with alterations in the above-mentioned network metrics.

\section{MATERIALS AND METHODS}

\section{Participants}

This study was part of a larger project on the vulnerability for new episodes in recurrent MDD (see Mocking et al (2016) for the project description). Inclusion criteria for rrMDD patients $(n=62)$ were: $(1) \geqslant 2 \mathrm{MDD}$ episodes according to the Structured Clinical Interview for DSM-IV Disorders (SCID), (2) current state of stable remission, defined as (i) a score of $\leqslant 7$ on the 17-item Hamilton Depression Rating Scale (HDRS) for $\geqslant 8$ weeks and (ii) no current depressive episode according to the SCID, (3) age between 35 and 65 years (to include a homogeneous age group and preclude conversion to bipolar disorder due to later experience of (hypo)manic episodes), (4) Dutch or English proficiency. Exclusion criteria for rrMDD patients were: (1) current diagnosis of alcohol/drug dependence, psychotic, or bipolar disorder, a predominant anxiety disorder or severe personality disorder according to the SCID, (2) MRI-incompatible implants or tattoos, (3) claustrophobia, (4) electroconvulsive therapy within 2 months before scanning, (5) history of seizure or head injury, (6) neurological disorder, (7) current severe physical illness, (8) use of psychoactive drugs/ medication $<4$ weeks before assessments. Incidental benzodiazepine use was allowed, but had to be terminated $>2$ days $(\geqslant 5$ half-lives) before assessments. HC $(n=41)$ were included when (i) they (according to the SCID) or their first-degree relatives did not have a lifetime diagnosis of psychiatric disorders and (ii) they met inclusion criteria 3 and 4 and did not meet exclusion criteria 2, 3, and 5-8. The samples were matched for sex, age, educational level, socioeconomic status, ethnicity, and handedness. Participants were recruited from previous studies and primary and secondary mental health-care institutes and through advertisements in online and house-to-house papers and posters in public places. Written informed consent was obtained and the study was approved by the Medical Ethical Committee of the Academic Medical Center.

\section{Experience Sampling Methodology}

Participants received an ESM palmtop before the scanning session (median $=13$ days; interquartile range $=14$ days) and were asked to fill out a short self-report questionnaire on affect, physical status, and context 10 times a day for 6 days. Beeps fell randomly in 10 90-min time blocks between 0730-2230 hours. Participants were instructed to complete the questionnaire within $15 \mathrm{~min}$. For the current study, we selected all variables related to mood and cognition (see Mocking et al (2016) for the complete ESM protocol, including-among others-items on company and daily 
events), including four positive mood items (enthusiastic, cheerful, relaxed, satisfied), seven negative mood items (agitated, anxious, down, irritated, lonely, guilty, restless), two positive cognition items (empowered, self-like), and four negative cognition items (ashamed, self-doubt, suspicious, worry) (see Supplementary Data S1 and Supplementary Table S1 for the content of the variables). Items were rated on a 7 -point Likert scale ranging from $1=$ 'not at all' to $7=$ 'very'. Ninety-seven participants completed the ESM protocol (57 rrMDD patients; $40 \mathrm{HCs}$ ). Next, we cleaned the data (see Supplementary Data S2 for details on the cleaning steps) and removed 903 observations from a total of 4466 observations, thereby excluding 28 participants (14 rrMDD patients; 14 HCs; see Supplementary Data S3 for differences in sample characteristics between included and excluded participants per group). This left a total of 3563 observations and 69 participants (43 rrMDD patients; $26 \mathrm{HCs}$ ) for ESM analyses. Subsequently, we checked whether variables contained substantial variation based on the visual inspection of boxplots. Three variables did not show substantial variation across participants: mood item 'anxious', mood item 'guilty', and cognition item 'suspicious' (Supplementary Data S4 and Supplementary Figure S1) and were excluded from further analyses. To capture affective instability, we calculated the mean-adjusted absolute successive difference (MAASD) per ESM variable and subject (Jahng et al, 2008, see Supplementary Data S5, S6 and S7, Supplementary Table S2 and Supplementary Figure S2 and S3 for details on the method and corresponding checks). Differences in MAASD between the rrMDD and $\mathrm{HC}$ group were calculated using an independent-samples Mann-Whitney $U$-test. Results with
Bonferroni-corrected $p$-values $\leqslant 0.004 \quad(0.05 / 14$ variables $)$ were considered significant.

\section{Image Preprocessing}

For details on the image acquisition parameters, preprocessing and postprocessing steps, and scrubbing, see Supplementary Data S8, S9 and S10. After image processing, 13 participants (9 rrMDD patients; 4 HCs) were excluded because of anatomical abnormalities $(n=5)$, excessive scrubbing (viz removal of $\geqslant 1 / 3$ of the volumes) $(n=6)$, or preprocessing failures $(n=2)$. This left a total of 90 participants (53 rrMDD patients; $37 \mathrm{HCs}$ ) for fMRI analyses.

\section{Graph Theory}

Network construction. As previously described in Servaas et al (2015), nodes were built by creating a sphere of $5 \mathrm{~mm}$ radius around 270 coordinates (Power et al, 2011), including bilateral amygdala, hippocampus, and caudate. The coordinates for these latter regions were determined using the Harvard-Oxford Subcortical Structural Atlas (80\% probability). No overlap was observed between the additional ROIs and the ROIs of Power et al (2011). Next, a whole-brain group mask was built based on the EPI images to locate the parts of the brain, which are free from susceptibility artifacts in all participants. Subsequently, the overlap was calculated voxel-wise between all nodes and the group mask. When a node overlapped $<50 \%$ with the group mask, it was excluded from further analysis. This was the case for 45 nodes. Next, we constructed a connectivity matrix per

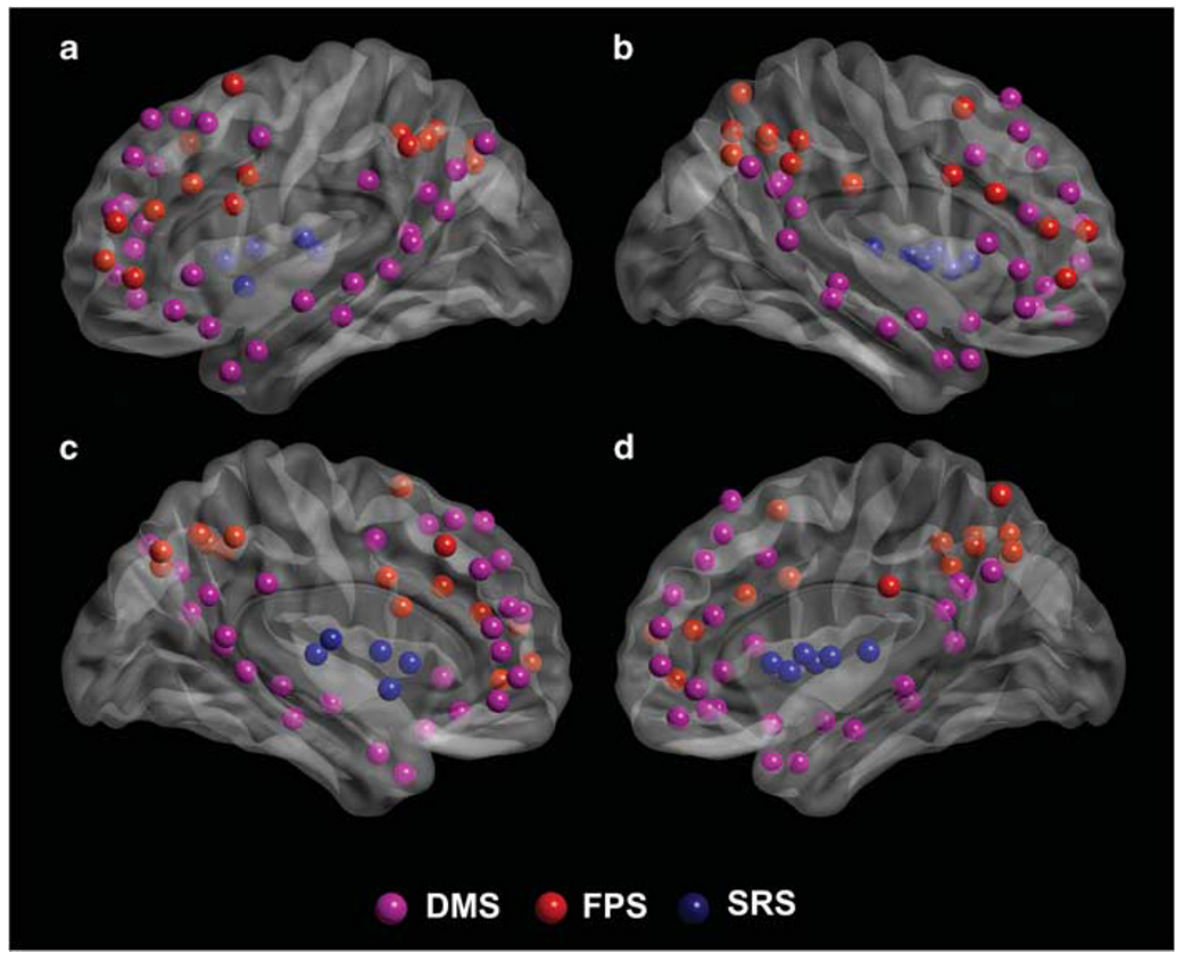

Figure I Module decomposition. Nodes could be partitioned in six functional subnetworks with a maximum number of within-group edges and a minimum number of between-group edges. In the current study, we focused on the default mode subnetwork (DMS, purple), frontoparietal subnetwork (FPS, red) and the salience/reward subnetwork (SRS, blue). Nodes are pasted on a surface template of the human brain using BrainNet Viewer (Xia et al, 20 I3). In the panels, different views are shown: (a) left lateral, (b) right lateral, (c) left medial, and (d) right medial. 
participant by extracting the regional mean time series for each of the remaining 225 nodes and calculated Pearson's correlations between all pairs. Furthermore, to prevent biases due to shared non-biological signal between adjacent nodes, correlations were set to zero when the distance was $<20 \mathrm{~mm}$ between the centers of two nodes (Power et al, 2011). In addition, correlations on the diagonal of the connectivity matrix and negative correlations were set to zero as well.

Thresholding and decomposition. We applied a range of proportional thresholds to each correlation matrix per participant to separate relevant from irrelevant edges. The threshold values ranged from 1 to $30 \%$ in increments of $1 \%$. Network measures were calculated on weighted graphs across the selected range of threshold values. Subnetworks were derived from the whole-brain graph by applying the algorithm of Blondel et al (2008) and the modularity finetuning algorithm of Sun et al (2009). For this procedure, we selected a single optimal threshold by using the method of Geerligs et al (2015). The optimal threshold in the current study was $1.35 \%$. In total, six subnetworks were derived with a maximum number of within-group edges and a minimum number of between-group edges (Rubinov and Sporns, 2010). These included the affective, DMS, FPS, somatosensory-motor, salience/reward (SRS), and visual subnetworks (Supplementary Data S11 and Supplementary Figure S4). For the analysis, we focused on the DMS, FPS, and SRS (Figure 1). The reason that we limited the results to these three subnetworks, as indicated in the introduction, was that they have most consistently been related to depression (Hamilton et al, 2013; Mulders et al, 2015). Furthermore, we sought to limit the number of tests in the statistical analyses.

Calculation of network measures and statistical analysis. Network measures were calculated on weighted graphs using functions implemented in the Brain Connectivity Toolbox (Rubinov and Sporns, 2010). Averaged across nodes, we calculated local efficiency and the participation coefficient per subnetwork. Local efficiency is calculated as the average inverse shortest path length between the neighbors of a specific node and the participation coefficient is calculated as the ratio of intra- vs intermodular connections per node (for an explanation of these measures, see Rubinov and Sporns (2010); the selection of these measures was based on Power et al (2011), wherein the authors investigated the compartmentalization (ie, segregation) and diversity (ie, integration) of relationships in subnetworks by using local efficiency and the participation coefficient, and we applied this method in the following previous papers Geerligs et al (2015) and Servaas et al $(2015,2016)$ to specifically investigate information processing within and between subnetworks; for a graphical explanation of the measures, see Supplementary Data S12 and Supplementary Figure S5). In other words, local efficiency is a measure of integration among the neighbors of a node. High local efficiency means that a node is part of a highly connected environment. Low local efficiency means that the node is part of a sparsely connected environment. The participation coefficient measures the extent to which a node connects to other nodes that are part of a different subnetwork. High participation coefficients mean that a node is mostly connected to nodes of a variety of other subnetworks. Low participation coefficients mean that a node is mostly connected to nodes of the same subnetwork (Power et al, 2011). Across the selected range of threshold values, we calculated (i) mean differences between the rrMDD and HC group per network measure for the DMS, FPS, and SRS, (ii) Pearson's correlations between the MAASD of ESM variables that showed significant group differences, and network measures for the DMS, FPS, and SRS in the rrMDD group only $(n=39)$. The latter was not calculated in the HC group, as the MAASD of the ESM variables did not show substantial variation (Supplementary Data S13 and Supplementary Figure S6). To obtain summarized scalars that are independent of single threshold selection, we applied the area under the curve (AUC) and threshold-free cluster enhancement (TFCE; Smith and Nichols, 2009) method across threshold values per network measure. The AUC gives an overall measure of significance across threshold values, whereas the TFCE method gives a measure of significance per threshold value (corrected for the number of threshold values). Next, nonparametric permutation testing was applied on the AUC and TFCE per network measure to assess whether results could have occurred by chance. To this end, group membership/MAASD values was/were permuted randomly and both difference measures were recalculated. This procedure was repeated 5000 times and a two-tailed test of the null hypothesis $(p<0.05)$ was performed.

\section{RESULTS}

\section{Sample Characteristics}

No significant differences were observed between rrMDD patients and $\mathrm{HC}$, except for residual symptomatology as measured by the HDRS (rrMDD patients $>\mathrm{HC}$; Table 1). When differences were recalculated for the selected samples used in the ESM and fMRI analyses, no significant differences were observed between rrMDD patients and HCs, except for employment status in the fMRI sample (employment status: rrMDD patients $<$ HCs; $\chi^{2}(2, N=90)$ $=6.13, p=0.047)$ and residual symptomatology in both samples (residual symptomatology: rrMDD patients $>\mathrm{HCs}$; ESM: $U=312.00, p=0.002$; fMRI: $U=540.00, p<0.0001)$.

\section{Main Effect of Group on Affective Instability}

The MAASD of the negative mood/cognition variables agitate, down, irritate, restless, and worry showed significant differences between rrMDD patients and HCs (rrMDD patients $>\mathrm{HCs}$; Figure 2 and Table 2). No significant differences in MAASD were observed between the two groups for positive mood/cognition variables and the other negative mood/cognition variables. Although the focus of the current study is on affective instability, it is notable that significant differences were found between rrMDD patients and HCs for the median of the positive $\mathrm{mood} /$ cognition variables enthusiastic, cheerful, relaxed, satisfied, and empowered (rrMDD patients $<$ HCs; see Supplementary Data S14 and Supplementary Table S3)

\section{Results on Network Measures}

Main effect of group. We found that the participation coefficient was decreased in the DMS in rrMDD patients 
Table I Sample Characteristics

\begin{tabular}{|c|c|c|c|c|c|c|}
\hline & \multirow[t]{2}{*}{$\operatorname{rrMDD}(n=62)$} & \multirow[t]{2}{*}{ HC $(n=4 I)$} & \multicolumn{4}{|c|}{ Between-group statistics } \\
\hline & & & $\chi^{2}$ & $T$ & $U$ & $p$ \\
\hline Age (years; mean (SD)) & $53.7(7.9)$ & $51.8(8.1)$ & & 1.17 & & 0.25 \\
\hline Education levels ${ }^{\mathrm{a}}$ & $0 / 0 / 0 / 4 / 21 / 23 / 14$ & $0 / 0 / 0 / 1 / 16 / 17 / 7$ & 1.49 & & & 0.69 \\
\hline Employment status levels ${ }^{c}$ & $24 / 23 / 15 / 0$ & $21 / 16 / 4 / 0$ & 3.70 & & & 0.16 \\
\hline Handedness levels ${ }^{d}$ & $4 / 50 / 4$ & $2 / 33 / 4$ & 0.44 & & & 0.80 \\
\hline Age of onset (years; mean (SD)) & $27.18(11.18)^{\mathrm{e}}$ & - & & & & - \\
\hline Episodes (mean (SD)) & $8.02(11.7)^{\mathrm{e}}$ & - & & & & - \\
\hline HDRS (mean (SD), range) & 2.81 (2.36), 0-9 & $1.02(1.42), 0-5$ & & & 686 & $<0.001$ \\
\hline
\end{tabular}

compared with HCs. No significant group differences were observed for local efficiency or the participation coefficient calculated for the SRS and FPS (Table 3).

Association between affective instability and network measures in rrMDD patients. For MAASD mood down, a significant negative correlation was found with the participation coefficient in the SRS. For MAASD mood irritate, a significant positive correlation was found with local efficiency in the FPS (Supplementary Data S15 and Supplementary Figures S7 and S8). No significant correlations were observed for the mood variables agitate and restless, and the cognition variable worry (Table 4).

Reanalyzing the data using binary graphs led to the same conclusions (Supplementary Data S16, Supplementary Tables S4, S17, and Supplementary Table S5). Furthermore, bootstrapping performed on the correlation slopes showed that the associations between affective instability and networks measures are fairly stable (Supplementary Data S18, Supplementary Figure S9 and S10). Moreover, a factor analysis on the MAASD data was performed to investigate the association between composite scores of the ESM data (instability in negative and positive affect) and network measures in rrMDD patients (see Supplementary Data S19, Supplementary Tables S6, S7, and Supplementary Figure S11). No significant associations were found, only a trend significant positive correlation between local efficiency in the DSM and factor 1 instability in negative affect $(p=0.080)$.

\section{DISCUSSION}

We investigated associations between affective instability and connectomics in functional subnetworks in rrMDD patients. For the ESM analysis, we found increased affective instability in most negative mood/cognition variables in rrMDD patients compared with HCs. For the graph analysis, we found that the DMS has less connections with other subnetworks in rrMDD patients compared with HCs. For the ESM-fMRI analysis, we observed highly specific associations between affective instability and network measures. rrMDD patients, who showed more instability in feeling down, had less connections between the SRS and other subnetworks. Furthermore, rrMDD patients, who showed more instability in feeling irritated, had higher local efficiency coefficients in the FPS.

\section{Affective Instability}

We found that rrMDD patients, compared with HCs, were temporally less stable in worrying and feeling down, agitated, irritated, and restless. Our findings are in line with ESM research showing (i) increased reactivity in negative affect to social stress (van Winkel et al, 2015) and stressful events (Husky et al, 2009), and (ii) increased negative affect on stressful days (O'Hara et al, 2014) in rMDD patients compared with HCs. The current study is the first ESM study in rrMDD patients, as patients in the above-mentioned studies did not suffer from multiple episodes or this was unspecified. Although a positive relationship has been found between reactivity of negative affect to negative external events and instability in negative affect in ESM studies, caution is warranted because (i) reactivity does not fully explain the level of instability, (ii) we only focused on mood in the current study, leaving out daily events and (iii) results with regard to reactivity in MDD in naturalistic settings have been inconsistent (Thompson et al, 2012). However, a recent meta-analysis has shown an association between lower psychological well-being and increased affective instability in MDD patients (Houben et al, 2015). The fact that we found increased affective instability in rrMDD patients may 


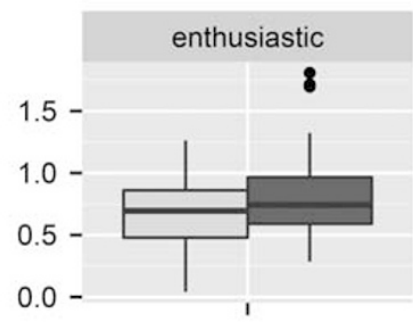

Boxplots MAASD ESM variables
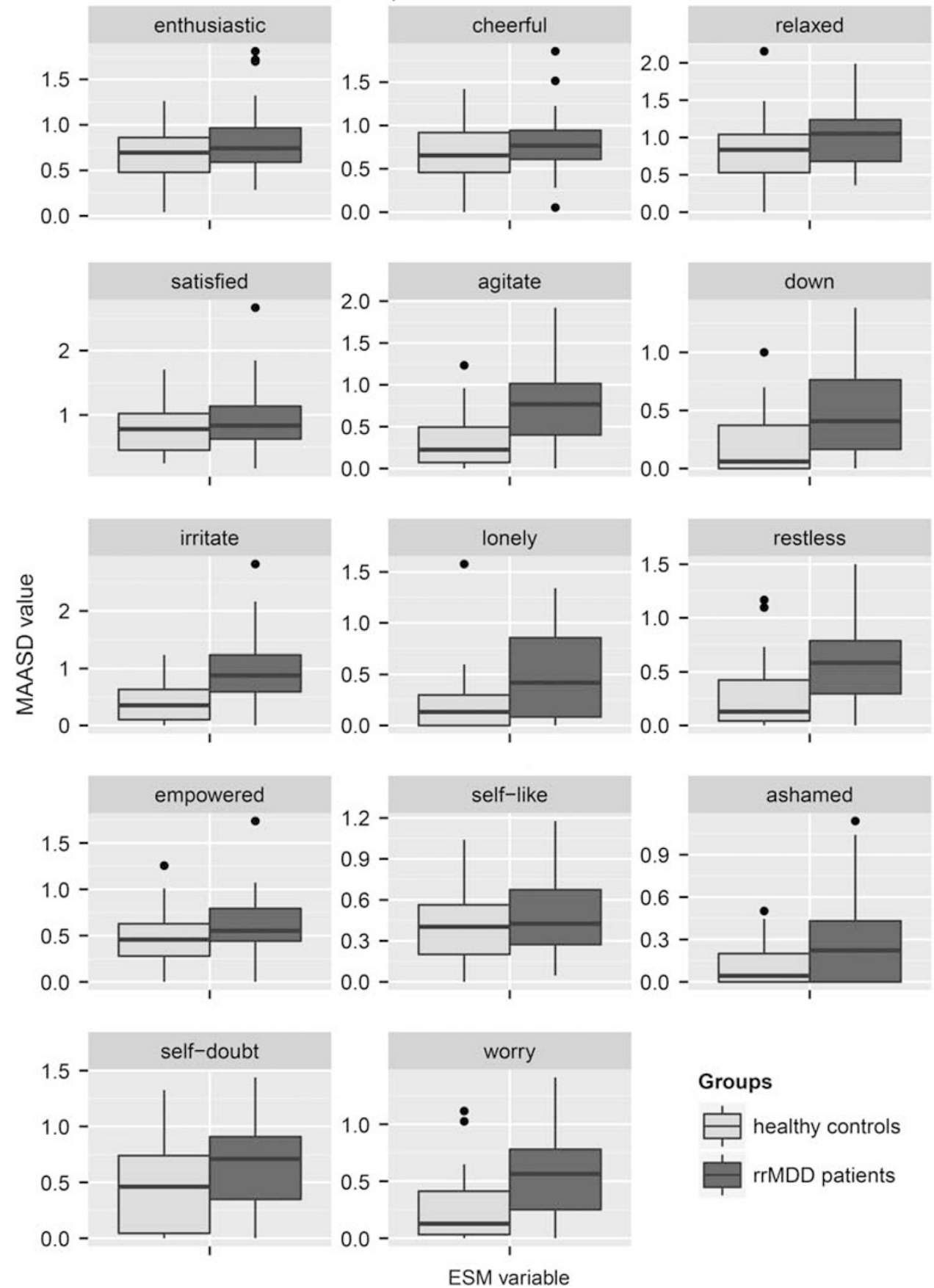

\section{Groups

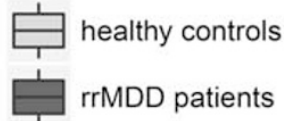

Figure 2 Boxplots of the MAASD for each ESM variable per group. ESM. experience sampling methodology; MAASD, mean adjusted absolute successive difference; rrMDD, remitted recurrent major depressive disorder.

indicate that this represents a trait effect rather than a state effect, although more research in rrMDD patients is needed to confirm the latter proposition.

The experience of positive affect was as temporally stable in rrMDD patients as in HCs, but was overall lower in rrMDD patients. In line with this, Knowles et al (2007) did not find differences in the degree of fluctuations in positive affect between rMDD patients and HCs, although neither for negative affect. Furthermore, van Winkel et al (2015) did not find significant differences in reactivity in positive affect to various stressors between rMDD patients and HCs; however, O'Hara et al (2014) found lower positive affect on stressful days between these two groups. It is also in line with research in MDD wherein no differences in instability of positive affect were found in patients compared with HCs, only lower levels of positive affect (Peeters et al, 2006; Thompson et al, 2012). Notably, different methods were used in the abovementioned studies, which should be kept in mind when interpreting their findings. O'Hara et al (2014) and van Winkel et al (2015) used multilevel models to predict mood 
Table 2 Main effect of Group on Affective Instability

\begin{tabular}{|c|c|c|c|}
\hline \multirow[t]{2}{*}{ ESM variable } & \multicolumn{3}{|c|}{ Between-group statistics } \\
\hline & $u$ & z & $p$ \\
\hline \multicolumn{4}{|l|}{ Positive mood } \\
\hline Enthusiastic & 472 & -1.31 & 0.189 \\
\hline Cheerful & 486 & -1.14 & 0.253 \\
\hline Relaxed & 449 & -1.59 & 0.111 \\
\hline Satisfied & 511 & -0.83 & 0.402 \\
\hline \multicolumn{4}{|l|}{ Negative mood } \\
\hline Agitate & 343.5 & -2.88 & $0.004 *$ \\
\hline Down & 346.5 & -2.84 & $0.004 *$ \\
\hline Irritate & 314 & -3.23 & $0.001 *$ \\
\hline Lonely & 351 & -2.80 & 0.005 \\
\hline Restless & 333.5 & -3.00 & $0.003 *$ \\
\hline \multicolumn{4}{|l|}{ Positive cognition } \\
\hline Empowered & 505 & -0.91 & 0.362 \\
\hline Self-like & 510 & -0.85 & 0.395 \\
\hline \multicolumn{4}{|l|}{ Negative cognition } \\
\hline Ashamed & 490 & -1.11 & 0.266 \\
\hline Self-doubt & $4 \mid 3.5$ & -2.03 & 0.043 \\
\hline Worry & 329 & -3.06 & $0.002 *$ \\
\hline
\end{tabular}

Abbreviations: ESM, experience sampling methodology; p, p-value; U, MannWhitney $U$ non-parametric test statistic; z, z-score.

The total sample size for the ESM analyses was $n=69$ (rrMDD patients: $n=43$; HCs: $n=26$ ).

*p-value $\leqslant(0.05 / 14=) 0.004$

Table 3 Main Effect of Group on Network Measures

\begin{tabular}{|c|c|c|c|c|}
\hline \multirow[t]{2}{*}{ Subnetwork } & \multicolumn{3}{|c|}{ Weighted } & \multirow[t]{2}{*}{ Direction } \\
\hline & $\begin{array}{c}\text { AUC } \\
\text { (P-value) }\end{array}$ & & $\begin{array}{l}\text { CE (threshold } \\
\text { values) }\end{array}$ & \\
\hline \multicolumn{5}{|l|}{ Local efficiency } \\
\hline DMS & 0.145 & - & & \\
\hline FPS & 0.363 & - & & \\
\hline SRS & 0.205 & - & & \\
\hline \multicolumn{5}{|c|}{ Participation coefficient } \\
\hline DMS & $0.026 * *$ & & $0.2-0.30 * *$ & $\mathrm{rrMDD}<\mathrm{HC}$ \\
\hline FPS & 0.587 & - & & \\
\hline SRS & 0.142 & - & & \\
\hline
\end{tabular}

Abbreviations: AUC, area under the curve; DMS, default mode subnetwork; FPS, frontal-parietal subnetwork; HC, healthy controls; rrMDD, remitted recurrent major depressive disorder, SRS, salience/reward subnetwork; TFCE, thresholdfree cluster enhancement.

The total sample size for the fMRI analyses was $n=90$ (rrMDD patients: $n=53$; HCs: $n=37)$.

*** $p$-value $<0.05$ state/reactivity from the group $\times$ stress interaction. Knowles et al (2007) used within-participant standard deviations. Notably, the MAASD is a more comprehensive measure to capture fluctuations in affect than the standard deviation, as temporal dependency (besides variability) is taken into account. This may explain the null findings in the study of Knowles et al (2007).

Several possible explanations have been proposed in the literature for increased affective instability in MDD patients, which may also have a role in rrMDD patients, including interpersonal impairment, difficulties in emotion regulation, and increased cognitive reactivity (O'Hara et al, 2014; Thompson et al, 2011, 2012). Furthermore, decreased positive affect may weaken personal resources and adaptive coping, as positive affect acts as a resilience factor (O'Hara et al, 2014). It would be of particular interest to investigate whether affective instability in negative affect predicts recurrence in MDD in future research. If this is the case, stabilizing negative affective responses to stress and increasing positive affect may represent clinical objectives to prevent relapse and guide decision-making for the implementation of recurrence preventive strategies.

\section{Connectomics}

We found that the DMS has less connections with other subnetworks in rrMDD patients compared with HCs, possibly leading to a more isolated position of this subnetwork within the network organization. The DMS has been reported to be more active during conditions of rest and is postulated to subserve functions, such as autobiographical memory, self-reflection, introspection, and emotion regulation (Buckner et al, 2008). Furthermore, numerous studies have shown the involvement of the DMS in rumination; an important feature in depressive states and an established risk factor for recurrence of MDD (Hamilton et al, 2011; Marchetti et al, 2012). Markedly, a theoretical model of the underlying neural mechanisms of rrMDD was constructed based on the DMS, postulating that most of its dysregulations observed in the acute phase are still present during remission (Marchetti et al, 2012). Specifically, it is proposed that ineffective switching between the DMS and task positive subnetwork-involved in external attention/cognition-increases the risk for recurrence (Marchetti et al, 2012). Thus far, connectomic studies in MDD have primarily been focused on within-subnetwork integration and showed increased regional connectivity in the DMS in patients compared with HCs (Gong and He, 2015). In our sample of rrMDD patients, we did not find increased local efficiency in

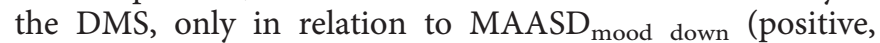
$p=0.053)$. Furthermore, we did not find significant differences in the network metrics for the SRS and FPS. It is possible that, during remission, these measures are normalized or influence mood and behavior in a more complex way. Future research should address which brain areas have altered connectivity with the DMS, and whether recurrence can be prevented by normalizing these abnormalities. For instance, a study, in which transcranial magnetic stimulation (TMS) is used, showed a reduction of depression-related hyperconnectivity in the DMS and an induction of anticorrelated connectivity between the DMS and FPS after 5 weeks of treatment (Liston et al, 2014). 
Table 4 Correlation Between Affective Instability and Network Measures in rrMDD Patients

\begin{tabular}{|c|c|c|c|}
\hline \multirow[t]{2}{*}{ Subnetwork } & \multicolumn{2}{|c|}{ Weighted } & \multirow[t]{2}{*}{ Direction } \\
\hline & $\begin{array}{c}\text { AUC } \\
\text { (p-value) }\end{array}$ & $\begin{array}{l}\text { TFCE (threshold } \\
\text { values) }\end{array}$ & \\
\hline
\end{tabular}

\begin{tabular}{ll}
\hline Mood agitate & \\
Local efficiency & \\
DMS & 0.110 \\
FPS & 0.19 \\
SRS & 0.714 \\
Participation coefficient & \\
DMS & 0.166 \\
FPS & 0.26 \\
SRS & 0.678
\end{tabular}

$\begin{array}{llcl}\begin{array}{l}\text { Mood down } \\ \text { Local efficiency }\end{array} & & & \\ \text { DMS } & 0.053 * & 0.01-0.06,0.09-0.11 * * & \text { Positive } \\ \text { FPS } & 0.393 & - & \\ \text { SRS } & 0.754 & - & \\ \text { Participation coefficient } & & - & \\ \text { DMS } & 0.161 & - & \text { Negative } \\ \text { FPS } & 0.371 & 0.02-0.30 * * & \end{array}$

$\begin{array}{llcc}\begin{array}{l}\text { Mood irritate } \\ \text { Local efficiency }\end{array} & & & \\ \text { DMS } & 0.250 & - & \\ \text { FPS } & 0.031 * * & 0.01-0.30 * * & \text { Positive } \\ \text { SRS } & 0.169 & - & \\ \text { Participation coefficient } & & - & \\ \text { DMS } & 0.237 & 0.23-0.30 * * & \text { Negative } \\ \text { FPS } & 0.066 * & - & \\ \text { SRS } & 0.796 & & \end{array}$

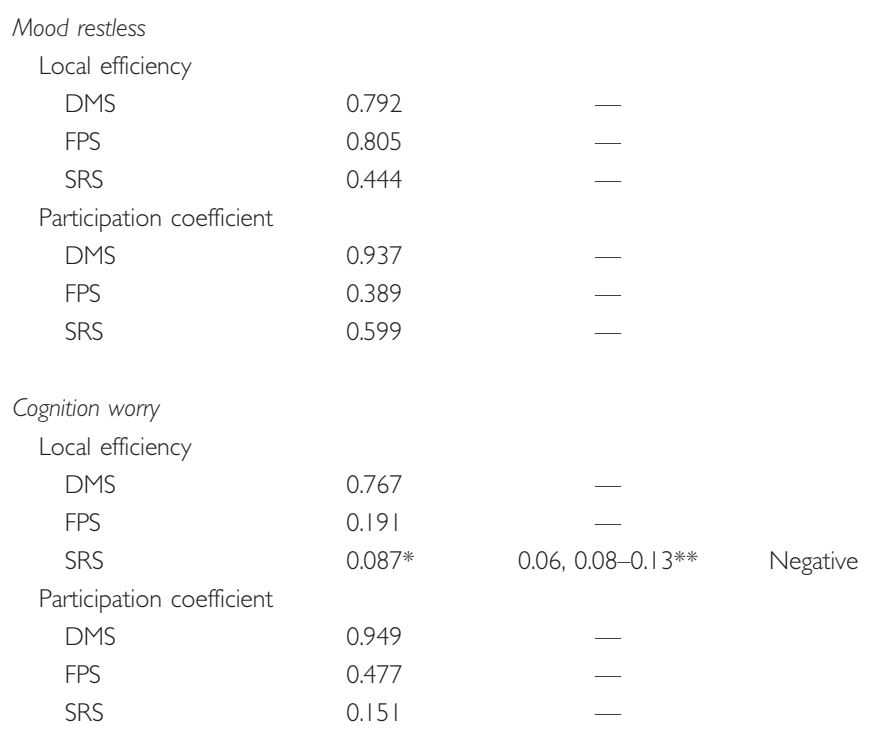

Abbreviations: AUC, area under the curve; DMS, default mode subnetwork; FPS, frontal-parietal subnetwork; rrMDD, remitted recurrent major depressive disorder; SRS, salience/reward subnetwork; TFCE, threshold-free cluster enhancement. The total sample size for the ESM-fMRI analyses was $n=39$ rrMDD patients.

*** $p$-value $<0.05$, ${ }^{p} p$-value $<0.10$.

\section{Affective Instability and Connectomics}

First, we found that rrMDD patients, who showed more instability in feeling down, had less connections between the SRS and other subnetworks. In the current study, the SRS consisted mainly of brain regions that are part of the striatum. Previous research has shown reduced striatum activation during reward processing in MDD patients compared with HCs (Pizzagalli et al, 2009; Smoski et al, 2009), which has also been found in recovered MDD patients (McCabe et al, 2009). Furthermore, computational models of reinforcement learning have shown impairments in MDD involving-among others-the striatum (Chen et al, 2015). Moreover, altered frontostriatal connectivity has been found in response to monetary gains and losses in unmedicated MDD patients compared with HCs (Admon et al, 2015b). The authors interpreted these results as an impairment in the positive and negative feedback circuit, leading to altered saliency of positive and negative events (Admon et al, 2015b). In rrMDD patients compared with HCs, the same authors found increased connectivity between the striatum and amygdala/hippocampus in response to mild stressors (Admon et al, 2015a). Thus, the results of the current study may suggest that altered integration between the SRS and other subnetworks is possibly associated with dysfunctions in depression-related processes, such as reward and stress.

Second, we found that rrMDD patients, who showed more instability in feeling irritated, had higher local efficiency coefficients in the FPS. Irritability has been shown to be prevalent in MDD and longitudinal studies in non-clinical samples have shown that irritability-related traits predict depressive and anxious psychopathology later in life (Leibenluft and Stoddard, 2013). Leibenluft (2011) proposed in her neurobiological model that ineffective frontal inhibition of emotional systems leads to feelings of irritation and frustration, when goals cannot be attained. In line with this, the FPS has been found to be involved in functions related to cognitive control (Laird et al, 2011). Indeed, two studies that induced irritability by recalling autobiographical experiences found increased prefrontal activation during irritability scripts compared with neutral scripts in HCs (Cerqueira et al, 2010, 2014). These findings suggest that our result of higher local efficiency in the FPS may act as a compensatory mechanism to control cognitively (fluctuating) feelings of irritation and frustration. Notably, it would be interesting to investigate whether the above-mentioned ESM-fMRI findings are specific to rrMDD patients or can also be found in HCs when, for example, they are under more stress due to negative life events or score higher on personality traits such as neuroticism, and show more affective instability.

To prevent first or recurrent depressive episodes, the idea has been coined to strengthen network connectivity underlying resilience (a.o. DMN: $\downarrow$; SRS: $\uparrow$; FPN: $\uparrow$; DMN-FPN anticorrelations: $\uparrow$ ) through interventions such as mindfulness, psychotherapy, and TMS. The results of our study are in line with pursuing these suggestions, but the effect of such interventions on connectomic measures/connectivity still needs to be demonstrated. With regard to TMS, multiple biomarkers have been identified that predict clinical outcome after repetitive TMS (rTMS), but there is still substantial heterogeneity in the biomarker type and effect. Furthermore, studies are limited that investigate whether rTMS induces 
(lasting) plasticity changes in subnetworks. Although promising, more research on the clinical applicability of functional connectivity in depression is needed to clarify these matters (see Fischer et al, 2016 for a recent review).

\section{Limitations}

The results from the ESM-fMRI analysis should be considered exploratory, because of the number of statistical tests that were performed. We tried to alleviate the multiple comparison problem by calculating the AUC and a mean of the nodal network measures per subnetwork. To provide insight into the reliability of the results, we performed permutation testing and bootstrapping (Supplementary Figure S18). Notably, it is difficult to correct adequately for multiple comparisons in graph analyses, as network measures are not independent of each other. Multivariate methods would be an option, but results from these types of analysis are more difficult to interpret (Simpson et al, 2013). For the above-mentioned reasons, our results are in need of replication.

\section{CONCLUSION}

The aim of the current study was to investigate associations between affective instability and connectomics in functional subnetworks in rrMDD patients. We found that $\operatorname{rrMDD}$ patients, compared with HCs, are less stable in their negative affect and that these dynamics are related to the way information is processed within- and between-specific functional subnetworks. The findings provide (i) real-life validity to connectomics using ESM and (ii) a neurobiological correlate associated with affective instability using connectomics. The findings may facilitate a better understanding of how fluctuations in real-life mood are represented in the brain of rrMDD patients, providing insights in the vulnerability profile of MDD.

\section{FUNDING AND DISCLOSURE}

We acknowledge the financial contribution of the iLab of the Department of Psychiatry of the UMCG (http://ilabpsychiatry.nl) to the appointment of MNS. MW is supported by the Dutch Brain Foundation (Hersenstichting Nederland: 2012(1)-03). LG is supported by a Rubicon grant from the Netherlands Organization for Scientific Research. The current study is supported by unrestricted personal grants from the AMC to RJTM (AMC PhD Scholarship) and CAF (AMC MD-PhD Scholarship), and a dedicated grant from the Dutch Brain Foundation (Hersenstichting Nederland: 2009(2)-72). HGR is supported by a NWO/ZonMW VENIGrant No. 016.126.059. This cohort study is registered under NTR3768. The funding sources had no involvement in the design and conduct of the study; collection, management, analysis, and interpretation of the data; preparation, review, or approval of the manuscript; and decision to submit the manuscript for publication. The authors declare no conflict of interest.

\section{REFERENCES}

Aan het Rot M, Hogenelst K, Schoevers RA (2012). Mood disorders in everyday life: a systematic review of experience sampling and ecological momentary assessment studies. Clin Psychol Rev 32: 510-523.

Admon R, Holsen LM, Aizley H, Remington A, Whitfield-Gabrieli S, Goldstein JM et al (2015a). Striatal hypersensitivity during stress in remitted individuals with recurrent depression. Biol Psychiatry 78: 67-76.

Admon R, Nickerson LD, Dillon DG, Holmes AJ, Bogdan R, Kumar P et al (2015b). Dissociable cortico-striatal connectivity abnormalities in major depression in response to monetary gains and penalties. Psychol Med 45: 121-131.

Bai F, Shu N, Yuan Y, Shi Y, Yu H, Wu D et al (2012). Topologically convergent and divergent structural connectivity patterns between patients with remitted geriatric depression and amnestic mild cognitive impairment. J Neurosci 32: 4307-4318.

Blondel VD, Guillaume J, Lambiotte R, Lefebvre E (2008). Fast unfolding of communities in large networks. J Stat Mech 10: P10008.

Buckner RL, Andrews-Hanna J, Schacter DL (2008). The brain's default network: anatomy, function, and relevance to disease. In: Kingstone A, Miller MB, Kingstone A, Miller MB (eds). The Year in Cognitive Neuroscience 2008. Blackwell Publishing: Malden, MA. pp 1-38.

Cerqueira CT, Almeida JR, Sato JR, Gorenstein C, Gentil V, Leite CC et al (2010). Cognitive control associated with irritability induction: an autobiographical recall fMRI study. Rev Bras Psiquiatr 32: 109-118.

Cerqueira CT, Sato JR, de Almeida JR, Amaro E Jr, Leite CC, Gorenstein C et al (2014). Healthy individuals treated with clomipramine: an fMRI study of brain activity during autobiographical recall of emotions. Transl Psychiatry 4: e405.

Chen C, Takahashi T, Nakagawa S, Inoue T, Kusumi I (2015). Reinforcement learning in depression: a review of computational research. Neurosci Biobehav Rev 55: 247-267.

De Raedt R, Koster EH (2010). Understanding vulnerability for depression from a cognitive neuroscience perspective: a reappraisal of attentional factors and a new conceptual framework. Cogn Affect Behav Neurosci 10: 50-70.

Fava GA, Visani D (2008). Psychosocial determinants of recovery in depression. Dialogues Clin Neurosci 10: 461-472.

Fischer AS, Keller CJ, Etkin A (2016). The applicability of functional connectivity in depression: pathways toward more targeted intervention. Bioll Psychiatry 1: 262-270.

Geerligs L, Renken RJ, Saliasi E, Maurits NM, Lorist MM (2015). A brain-wide study of age-related changes in functional connectivity. Cereb Cortex 25: 1987-1999.

Gong Q, He Y (2015). Depression, neuroimaging and connectomics: a selective overview. Biol Psychiatry 77: 223-235.

Groenewold NA, Opmeer EM, de Jonge P, Aleman A, Costafreda SG (2013). Emotional valence modulates brain functional abnormalities in depression: evidence from a metaanalysis of fMRI studies. Neurosci Biobehav Rev 37: 152-163.

Hamilton JP, Chen MC, Gotlib IH (2013). Neural systems approaches to understanding major depressive disorder: an intrinsic functional organization perspective. Neurobiol Dis 52: 4-11.

Hamilton JP, Furman DJ, Chang C, Thomason ME, Dennis E, Gotlib IH (2011). Default-mode and task-positive network activity in major depressive disorder: implications for adaptive and maladaptive rumination. Biol Psychiatry 70: 327-333.

Hardeveld F, Spijker J, De Graaf R, Nolen WA, Beekman AT (2010). Prevalence and predictors of recurrence of major depressive disorder in the adult population. Acta Psychiatr Scand 122: 184-191.

Houben M, Van Den Noortgate W, Kuppens P (2015). The relation between short-term emotion dynamics and psychological well-being: a meta-analysis. Psychol Bull 141: 901-930.

Husky MM, Mazure CM, Maciejewski PK, Swendsen JD (2009). Past depression and gender interact to influence emotional reactivity to daily life stress. Cogn Ther Res 33: 264-271.

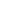


Jacobs RH, Jenkins LM, Gabriel LB, Barba A, Ryan KA, Weisenbach SL et al (2014). Increased coupling of intrinsic networks in remitted depressed youth predicts rumination and cognitive control. PLoS ONE 9: e104366.

Jahng S, Wood PK, Trull TJ (2008). Analysis of affective instability in ecological momentary assessment: Indices using successive difference and group comparison via multilevel modeling. Psychol Methods 13: 354-375.

Knowles R, Tai S, Jones SH, Highfield J, Morriss R, Bentall RP (2007). Stability of self-esteem in bipolar disorder: comparisons among remitted bipolar patients, remitted unipolar patients and healthy controls. Bipolar Disord 9: 490-495.

Laird AR, Fox PM, Eickhoff SB, Turner JA, Ray KL, McKay DR et al (2011). Behavioral interpretations of intrinsic connectivity networks. J Cogn Neurosci 23: 4022-4037.

Leibenluft E (2011). Severe mood dysregulation, irritability, and the diagnostic boundaries of bipolar disorder in youths. $\mathrm{Am} \mathrm{J}$ Psychiatry 168: 129-142.

Leibenluft E, Stoddard J (2013). The developmental psychopathology of irritability. Dev Psychopathol 25: 1473-1487.

Liston C, Chen AC, Zebley BD, Drysdale AT, Gordon R, Leuchter B et al (2014). Default mode network mechanisms of transcranial magnetic stimulation in depression. Biol Psychiatry 76: 517-526.

Marchetti I, Koster EH, Sonuga-Barke EJ, De Raedt R (2012). The default mode network and recurrent depression: a neurobiological model of cognitive risk factors. Neuropsychol Rev 22: 229-251.

McCabe C, Cowen PJ, Harmer CJ (2009). Neural representation of reward in recovered depressed patients. Psychopharmacology (Berl) 205: 667-677.

Mocking RJ, Figueroa CA, Rive MM, Geugies H, Servaas MN, Assies J et al (2016). Vulnerability for new episodes in recurrent major depressive disorder: protocol for the longitudinal DELTAneuroimaging cohort study. BMJ Open 6: e009510-2015-009510.

Mulders PC, van Eijndhoven PF, Schene AH, Beckmann CF, Tendolkar I (2015). Resting-state functional connectivity in major depressive disorder: a review. Neurosci Biobehav Rev 56: 330-344.

O'Hara RE, Armeli S, Boynton MH, Tennen H (2014). Emotional stress-reactivity and positive affect among college students: the role of depression history. Emotion 14: 193-202.

Peeters F, Berkhof J, Delespaul P, Rottenberg J, Nicolson NA (2006). Diurnal mood variation in major depressive disorder. Emotion 6: 383-391.

Pizzagalli DA, Holmes AJ, Dillon DG, Goetz EL, Birk JL, Bogdan R et al (2009). Reduced caudate and nucleus accumbens response to rewards in unmedicated individuals with major depressive disorder. Am J Psychiatry 166: 702-710.

Power JD, Cohen AL, Nelson SM, Wig GS, Barnes KA, Church JA et al (2011). Functional network organization of the human brain. Neuron 72: 665-678.

Qin J, Wei M, Liu H, Chen J, Yan R, Yao Z et al (2015). Altered anatomical patterns of depression in relation to antidepressant treatment: evidence from a pattern recognition analysis on the topological organization of brain networks. J Affect Disord 180: 129-137.
Rubinov M, Sporns O (2010). Complex network measures of brain connectivity: uses and interpretations. Neuroimage 52: 1059-1069.

Servaas MN, Geerligs L, Bastiaansen JA, Renken RJ, Marsman JC, Nolte IM et al (2016). Associations between genetic risk, functional brain network organization and neuroticism. Brain Imaging Behav E-pub ahead of print.

Servaas MN, Geerligs L, Renken RJ, Marsman JB, Ormel J, Riese H et al (2015). Connectomics and neuroticism: an altered functional network organization. Neuropsychopharmacology 40: 296-304.

Simpson SL, Bowman FD, Laurienti PJ (2013). Analyzing complex functional brain networks: Fusing statistics and network science to understand the brain. Statistics Surveys 7: 1-36.

Smith SM, Nichols TE (2009). Threshold-free cluster enhancement: addressing problems of smoothing, threshold dependence and localisation in cluster inference. Neuroimage 44: 83-98.

Smoski MJ, Felder J, Bizzell J, Green SR, Ernst M, Lynch TR et al (2009). fMRI of alterations in reward selection, anticipation, and feedback in major depressive disorder. J Affect Disord 118: $69-78$.

Sporns O (2012). Discovering the Human Connectome. The MIT Press: Cambridge, MA.

Steinert C, Hofmann M, Kruse J, Leichsenring F (2014). The prospective long-term course of adult depression in general practice and the community. A systematic literature review. J Affect Disord 152-154: 65-75.

Sun Y, Danila B, Josic K, Bassler KE (2009). Improved community structure detection using a modified fine-tuning strategy. Epl 86: 28004.

Thompson RJ, Berenbaum H, Bredemeier K (2011). Cross-sectional and longitudinal relations between affective instability and depression. J Affect Disord 130: 53-59.

Thompson RJ, Mata J, Jaeggi SM, Buschkuehl M, Jonides J, Gotlib IH (2012). The everyday emotional experience of adults with major depressive disorder: examining emotional instability, inertia, and reactivity. J Abnorm Psychol 121: 819-829.

Trull TJ, Solhan MB, Tragesser SL, Jahng S, Wood PK, Piasecki TM et al (2008). Affective instability: measuring a core feature of borderline personality disorder with ecological momentary assessment. J Abnorm Psychol 117: 647-661.

van Winkel $M$, Nicolson NA, Wichers $M$, Viechtbauer W, Myin-Germeys I, Peeters F (2015). Daily life stress reactivity in remitted versus non-remitted depressed individuals. Eur Psychiatry 30: $441-447$.

Verhage F (1964). Intelligentie en leeftijd onderzoek bij Nederlanders van twaalf tot zevenenzeventig jaar [Intelligence and age research with Dutch people aged twelve to seventyseven years]. Van Gorcum: Assen.

Widiger TA, Mangine S, Corbitt EM, Ellis CG, Thomas GV (1995). Personality Disorder Interview-IV. A Semistructured Interview for the Assessment of Personality Disorders. Professional Manual. Psychological Assessment Resources: Odessa, FL.

Xia M, Wang J, He Y (2013). BrainNet Viewer: a network visualization tool for human brain connectomics. PLoS ONE 8: e68910.

Supplementary Information accompanies the paper on the Neuropsychopharmacology website (http://www.nature.com/npp) 